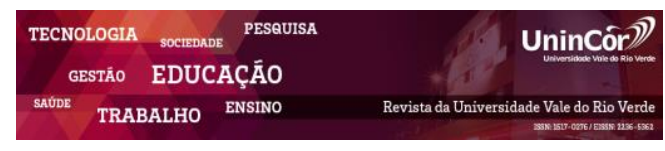

Revista da Universidade Vale do Rio Verde ISSN: 1517-0276 / EISSN: 2236-5362 v. $17 \mid$ n. 1 | Ano 2019

Débora Souza Monteiro

Graduação Odontologia - Universidade de Itaúna deboramonteiroodonto@hotmail.com

Mikaelle Queiroz de Morais

Graduação Odontologia - Universidade de Itaúna mikaelle_queiroz@hotmail.com

Regina Coeli Cançado Peixoto Pires

Doutora em Epidemiologia

Faculdade de Odontologia- Universidade de Itaúna rcppires@gmail.com

\section{LEVANTAMENTO EPIDEMIOLÓGICO DE CÁRIE DENTÁRIA EM CRIANÇAS DE 12 ANOS BONFIM-MG, 2017}

\section{RESUMO}

A cárie dentária é uma doença multifatorial, infecciosa, transmissível, dieta dependente que produz a desmineralização do esmalte dentário e acompanha a humanidade desde tempos imemoráveis. Medir essa doença na população é difícil, mas torna-se necessária, pois auxilia na elaboração de estratégias para implantação e avaliação de medidas para prevenção e controle. O objetivo deste estudo foi avaliar a cárie dentária na população de 12 anos em Bonfim, Minas Gerais; comparando os resultados com os dados nacional e regional como também relacionar à meta proposta pela Organização Mundial de Saúde(OMS). A pesquisa foi realizada em uma escola estadual, com população de estudo composta de 71 crianças. Trata-se de um estudo transversal e as crianças foram examinadas de acordo com a metodologia do SB Brasil 2010. Os dados foram tabulados utilizando o Software SB Dados, disponível no site do Ministério da Saúde. Foram geradas tabelas de frequência, análise descritiva e associações de variáveis. Os resultados mostraram que as crianças de 12 anos deste município apresentaram um CPO-D médio de 4,78, distante das metas da OMS para o ano de 2000 (CPO-D=3) e 2010 (CPO$\mathrm{D}=1$ ). A média de dentes cariados foi de 3,33 , de dentes perdidos 0,12 e de dentes obturados 0,81 . Observou-se grande diferença entre o CPO-D da região rural $(7,25)$ e urbana $(2,32)$. Concluiu-se que a realização do levantamento epidemiológico beneficiou o município, pois não existiam até o momento nenhum dado sobre a saúde bucal desta população, possibilitando o planejamento de medidas de prevenção e tratamento.

Palavras-chave: Cárie dentária. Epidemiologia. Saúde bucal. Escolares. Saúde pública.

\section{EPIDEMIOLOGICAL SURVEY OF DENTAL CAVITY IN 12-YEAR-OLD CHILDREN BONFIM-MG, 2017.}


to the SB Brasil 2010 methodology. The data were tabulated using the Software SB Dados, available on the Health Ministry website. Frequency tables, descriptive analysis and associations of variables were created. The results show that the 12 -year-old children from this county present a medium DMFT of 4,78, distant from the goals of WHO for the year of 2000 (DMFT=3) and $2010($ DMFT $=1)$. The average of decayed teeth was of 3,33, of lost teeth 0,12 and sealed teeth 0,81 . It was observed a large difference between the countryside $(7,25)$ and the urban region $(2,32)$. It was concluded that the realization of the epidemiological survey benefited the county, because there were until the moment no data about the oral health of this population, enabling the planning of prevention and treatment measures.

Keywords: Dental cavity, Epidemiological, Oral health, School children, Public health.

\section{Recebido em: 31/07/2018 - Aprovado em: 18/03/2019 - Disponibilizado em: 15/07/2019}

\section{INTRODUÇÂO}

A saúde bucal, na maioria dos municípios brasileiros, constitui ainda um grande desafio aos princípios do Sistema Único de Saúde, principalmente no que se refere à universalização e à equidade do atendimento.

A cárie dentária é uma doença multifatorial, infecciosa, transmissível, dieta dependente que produz a desmineralização do esmalte dentário e acompanha a humanidade desde tempos imemoráveis.

Dentro desse contexto, essa doença ainda se configura em um dos principais problemas de saúde bucal a serem equacionados. Entretanto, reduções significativas têm sido encontradas na prevalência e na severidade desta doença (GOMES et al.,2004).

Por esse motivo, medir o estado de saúde de uma população é uma tarefa complicada, porém, que se tornou necessária a fim de se elaborar diagnósticos e intervenções. Para observação da prevalência da doença de forma coerente, foram criados índices de estudo e compreensão de saúde bucal relacionado à cárie dentária. Foram eles o CPO-D e o ceo-d, os quais medem respectivamente, a prevalência de dentes cariados, perdidos e obturados na dentição permanente e decídua, oferecendo assim, uma média de comparação da situação encontrada (CARVALHO, 2008; CYPRIANO, 2005).

No Brasil, a epidemiologia em saúde bucal tem tido historicamente uma atuação pouco expressiva. Foram realizados apenas 5 levantamentos epidemiológicos (1986, 1993, 1996, 2003 e 2010), que mostraram redução no índice CPO-D na faixa etária de 12 anos. Em 1986 o CPO-D foi de 6,65 e em 2010 apresentou um resultado de 2,1. Durante décadas, a política de saúde bucal no Brasil foi centrada na prestação de assistência à doença, mas ainda uma grande parcela da população brasileira não tem acesso a cuidados odontológicos. Pela Portaria $n^{\circ}$ 1.444 de 28 de dezembro de 2000, o Ministério da Saúde estabeleceu incentivo financeiro para a reorganização da atenção à saúde bucal dos municípios (BRASIL, 2000). 
Diante do exposto, este estudo teve como objetivo avaliar a cárie dentária, através dos índices CPO-D e ceod na população de 12 anos na cidade de Bonfim, Minas Gerais, podendo assim auxiliar em programas de prevenção, tratamento e controle dessa doença. Segundo o Atlas de Desenvolvimento Humano 2010, esse município apresenta um IDH de 0,637 e população de 6.818 habitantes, sendo 48,87\% residente da zona urbana e $51,13 \%$ residente na zona rural.

\section{METODOLOGIA}

Trata-se de um estudo transversal e a coleta de dados foi realizada de acordo com a metodologia do Levantamento das Condições de Saúde Bucal da População Brasileira- SB Brasil 2010.

A população do estudo foi constituída por crianças com idade de 12 anos, procedentes de região rural e urbana, que estudavam na escola estadual no município de Bonfim, MG.

O projeto foi aprovado pelo Comitê de Ética em Pesquisa da Universidade de ItaúnaMG sob o número CAAE 8972417.0.0000.5144.

\section{RESULTADOS}

Participaram do estudo 71 escolares com idade de 12 anos.

Os exames foram realizados por dois examinadores calibrados através do Coeficiente de Kappa, com resultado de 0,92.

Na Tabela 1 é apresentada a distribuição da população em relação ao local de moradia, sendo que $43(60,6 \%)$ dos escolares residiam em região urbana e 29(39,4\%) em região rural.
Tabela 1: Distribuição dos escolares de 12 anos, segundo a região de moradia, Bonfim-MG, 2017.

\begin{tabular}{ccc}
\hline \hline Região & Frequência & Porcentagem \\
\hline Rural & 28 & 39,4 \\
Urbana & 43 & 60,6 \\
Total & 71 & 100,0 \\
\hline \hline
\end{tabular}

O CPO-D médio do município foi de 4,78 e relacionando esse índice nas regiões urbana e rural observou-se que para a população examinada da região rural o CPO-D foi de 7,25 e da região urbana encontrou-se um CPO-D de 2,32. Verificou-se que 12 alunos apresentavam CPO-D $=0$ e dentre aqueles com CPO-D $>3$ foram encontradas $7(9,8 \%)$ crianças que apresentaram CPO-D>10 (Tabela 2).

Tabela 2: Distribuição dos escolares de 12 anos, segundo o CPO-D, Bonfim-MG, 2017.

\begin{tabular}{ccc}
\hline \hline CPO-D & Frequência & Porcentagem \\
\hline 0 & 12 & 16,9 \\
1 & 11 & 15,5 \\
2 & 9 & 12,7 \\
3 & 6 & 8,5 \\
$>3$ & 33 & 46,4 \\
Total & 71 & 100,0 \\
\hline \hline
\end{tabular}

A Tabela 3 descreve o resultado do ataque de cárie na dentição permanente para a idade estudada. A média de dentes cariados, realizado através da divisão do número de dentes cariados pelo número de crianças, foi de 3,33 por escolar. Pode-se observar também que $16(22,6 \%)$ dos escolares estavam livres da cárie e $55(77,4 \%)$ apresentaram pelo menos um dente com lesão cariosa. 
Tabela 3: Distribuição do número de dentes cariados, dos escolares de 12 anos, Bonfim-MG, 2017.

\begin{tabular}{ccc}
\hline \hline $\mathrm{N}^{\circ}$ dentes cariados & Frequência & Porcentagem \\
\hline 0 & 16 & 22,6 \\
1 & 14 & 19,7 \\
2 & 7 & 9,9 \\
3 & 6 & 8,5 \\
$>3$ & 28 & 39,3 \\
Total & 71 & 100,0 \\
\hline \hline
\end{tabular}

Na Tabela 4, observa-se o número de dentes perdidos, o qual apresenta uma média de 0,12 por escolar, esta média foi obtida através da divisão do número de dentes perdidos pelo número de crianças. Foram encontrados 58 dentes restaurados e de acordo com a Tabela 5, $10(14,1 \%)$ escolares apresentaram apenas um dente restaurado (obturado).

Tabela 4: Distribuição do número de dentes perdidos dos escolares de 12 anos, Bonfim, 2017.

\begin{tabular}{ccc}
\hline \hline$N^{\circ}$ dentes perdidos & Frequência & Porcentagem \\
\hline 0 & 65 & 91,5 \\
1 & 4 & 5,6 \\
2 & 1 & 1,4 \\
3 & 1 & 1,4 \\
Total & 71 & 100,0 \\
\hline \hline
\end{tabular}

Tabela 5: Distribuição do número de dentes obturados, dos escolares de 12 anos, Bonfim-MG, 2017.

\begin{tabular}{ccc}
\hline \hline $\begin{array}{l}\mathrm{N}^{\circ} \text { dentes } \\
\text { obturados }\end{array}$ & Frequência & Porcentagem \\
\hline 0 & 45 & 63,4 \\
1 & 10 & 14,1 \\
2 & 6 & 8,5 \\
3 & 5 & 7,0 \\
4 & 4 & 5,6 \\
5 & 1 & 1,4 \\
Total & 71 & 100,0 \\
\hline \hline
\end{tabular}

Outra importante variável analisada, foi a necessidade de tratamento, que poderia ser: restauração de uma superfície, restauração de duas superfícies, necessidade de confecção de coroa, confecção de faceta estética, tratamento pulpar e exodontia. Na população do estudo foram encontrados 100 dentes que necessitavam de tratamento e dentre esses $78(78 \%)$ precisavam de restauração de uma superfície e 21(21\%) restauração de duas superfícies e apenas $1(1 \%)$ de tratamento pulpar. Um fator extremamente favorável é o fato de nenhuma criança necessitar da realização de exodontias.(Tabela 6).

Tabela 6: Necessidade de tratamento em relação ao número de dentes dos escolares residentes em Bonfim, 2017.

\begin{tabular}{ccc}
\multicolumn{3}{c}{2017.} \\
Necessidade de tratamento & $\begin{array}{c}\text { Número de } \\
\text { dentes }\end{array}$ & Porcentagem \\
\hline $\begin{array}{c}\text { Rest. 1 } \\
\text { superfície }\end{array}$ & 78 & $78 \%$ \\
Rest. 2 & & \\
superfícies & 21 & $21 \%$ \\
Tratamento & 1 & $1 \%$ \\
pulpar & 100 & $100 \%$ \\
Total &
\end{tabular}

\section{4-DISCUSSÃO}

A saúde Bucal é parte integrante e essencial da saúde geral e sendo assim, é um fator determinante para a qualidade de vida. Os agravos à saúde bucal são problemas de saúde pública porque tem um impacto significante no indivíduo e na comunidade, possuem alta prevalência e podem ser efetivamente prevenidos e controlados pela ação conjunta da comunidade, profissionais e indivíduos (MELO,2005). 
Um dos meios de se avaliar a saúde bucal é através da realização dos levantamentos epidemiológicos, os quais são importantes instrumentos para definição, implementação e avaliação de ações coletivas e individuais, preventivas e assistenciais. É uma forma de conhecer a realidade epidemiológica de determinada população, devendo ser realizado periodicamente (ANTUNES,2006).

No Brasil foram realizados apenas cinco levantamentos epidemiológicos, sendo que o primeiro, foi no ano de 1986 e é também denominado de levantamento da zona urbana, pois as pesquisas foram realizadas apenas nas capitais (16 capitais). Apresentou como resultado um CPO-D de 6,65 para a idade de 12 anos. Em 1993 realizou-se o segundo levantamento, entretanto foi financiado pelo SESI, e tinha como objetivo avaliar os serviços prestados por essa empresa na área de saúde bucal. Passados dez anos, realizou-se outro levantamento epidemiológico de amplitude nacional, e tinha como objetivo avaliar as alterações no perfil da população brasileira transcorridos dez anos. Este avaliou cárie dentária, em crianças de seis a doze anos de idade, que estudavam em escolas públicas e privadas. Apresentou como resultado um CPO-D de 3,06 na faixa etária de doze anos. Em 2003, foi feito o maior e mais amplo levantamento, este avaliou cárie dentária, doença periodontal, má oclusão, edentulismo, fluorose e lesões bucais. Teve como resultado um CPO-D de 2,78 na faixa etária de doze anos, onde $70 \%$ das crianças brasileiras e cerca de $90 \%$ dos adolescentes de quinze a dezenove anos apresentam pelo menos um dente permanente com experiência de cárie dentária. E por fim, em
2010 realizou-se o quinto levantamento epidemiológico, seguindo a mesma metodologia do de 2003, com a finalidade de permitir realizar comparações entre eles, e apresentou um CPO-D de 2,07 aos 12 anos ( RONCALLI, 2012).

Em contrapartida, na atualidade tem ocorrido um aumento considerável da realização de levantamentos epidemiológicos em nível municipal, e estes na sua grande maioria são realizados por docentes de cursos de odontologia, com o objetivo de conhecer o perfil epidemiológico da cárie dentária em sua cidade. $\mathrm{O}$ município de pequeno porte avaliado neste estudo foi Bonfim, que apresenta uma população de aproximadamente 6.818 habitantes, de acordo com o censo de 2010 e se localiza na região metropolitana de Belo horizonte.

Ao analisar os dados, observou-se que esse município apresentou um CPO-D aos 12 anos de 4,78, ou seja, cada criança apresenta aproximadamente cinco dentes cariados, perdidos ou obturados. Ao comparar esses dados com o SB Brasil 2010, o qual teve um CPO-D de 2,07 , verificou-se que o município apresentou um CPO-D desfavorável, sendo duas vezes maior que a média brasileira. Outra comparação importante, é a média de dentes cariados, que em Belo Horizonte-MG, capital do estado, no SB Brasil 2010 foi de 0,56, já no município de Bonfim foi de 3,33.

Deve-se também, comparar os resultados com o SB Minas Gerais, que em 2012 fez um levantamento epidemiológico das condições bucais pela Secretaria de Estado de Saúde de Minas Gerais. A amostral foi realizada em 60 municípios do interior, tendo sido examinadas 4.898 pessoas em todo o estado, pertencentes às 
faixas etárias de 5, 12, 15 a 19, 35 a 44 e 65 a 74 anos. Este estudo seguiu a mesma metodologia do SB Brasil 2010, para assim permitir a comparação dos resultados. A média do CPO-D aos 12 anos para o estado de Minas Gerais foi de 1,8 , sendo muito pouca acima da média da região sudeste que foi de 1,7 e abaixo da média brasileira que foi 2,1. (MINAS GERAIS, 2013). Comparando esses resultados com o CPO-D encontrado em Bonfim percebeu-se que nesse município o índice encontra-se muito maior.

Outro fator que deve ser levado em consideração, é a meta proposta pela Organização Mundial de Saúde (OMS), que determina , para a idade de 12 anos, um CPO-D menor que um para o ano de 2010. Também o CPO-D do município $(4,78)$ encontra-se muito longe desta meta e sendo classificado como de alta prevalência de cárie, por apresentar um valor entre 4,5 a 6,5(PINTO, 2000).

As cidades que apresentam níveis altos de cárie, normalmente também apresentam população com perfil de renda insuficiente, adultos sem alfabetização, menor renda familiar e menor Índice de Desenvolvimento Humano (IDH) (PERES, 2003).

$\mathrm{O}$ atlas de desenvolvimento humano, informa que Bonfim apresenta um IDH de 0,637, uma renda per capita média de quinhentos e doze reais e noventa e quatro centavos e a proporção de crianças de onze a treze anos de idade frequentando os anos finais do ensino fundamental é de $82,44 \%$. O grau de escolaridade, assim como a renda, podem ser considerados determinantes na escolha por estilos de vida mais saudáveis ou não, além de influenciar na percepção dos indivíduos sobre a sua saúde, bem como o alto cuidado (FIGUEIREDO,2006).

Há um predomínio do componente cariado, apresentando uma média de 3,33 dentes cariados por criança, em contrapartida, a média de dentes obturados é de 0,81 , este fato demonstrou que o acesso aos serviços para restaurar dentes continua sendo um desafio para a sociedade, aprofundando a contradição de um país onde há muitos dentistas, mas onde a população não consegue $\mathrm{o}$ acesso de forma igualitária aos serviços proporcionados por eles (NARVAI,2006).

Observou-se grande discrepância entre o CPO-D da região urbana $(2,32)$ com o da região rural $(\mathrm{CPO}-\mathrm{D}=7,25)$. Pode-se sugerir que o valor alto desse índice na zona rural possa estar relacionado a ausência de sistema de abastecimento de água prejudicando o uso de flúor sistêmico e uma dieta cariogênica com maior do consumo de sacarose (doces); além da deficiência na limpeza bucal.

Normalmente a população rural tem acesso a água por meio de poços artesianos, não tendo assim em sua composição o flúor. Nos dias atuais, sabe-se dos benefícios deste composto químico, que interfere na dinâmica do processo de desenvolvimento da cárie dentária, sendo de fundamental importância a sua presença constante na cavidade bucal, pois potencializa o processo de remineralização e reduz a desmineralização. Em relação à dieta cariogênica a população rural tem um maior costume de produzir doces, apresentando um maior consumo. Sabe-se que a sacarose é utilizada pelas bactérias do biofilme para seu metabolismo energético, aumentando assim a chance de se 
desenvolver lesões cariosas (SANTOS,2011;

LEITES,2006) .

$\mathrm{O}$ alto valor do índice CPO-D mostrou que é preciso adaptar as politicas públicas do município, para assim melhorar a saúde bucal da desta população. Frente a isso pode-se ampliar as equipes de saúde bucal, visto que no município existem apenas três dentistas que fazem atendimento nas Unidades Básicas de Saúde (UBS), aumentar o vínculo das agentes de saúde, pois estes profissionais realizam as visitas domiciliares fazendo o cadastro da população, aproximando a comunidade do atendimento odontológico e podem transmitir informações importantes sobre a saúde bucal.

\section{5-CONCLUSÃO}

A realização deste levantamento epidemiológico foi de extrema importância, pois através da análise dos dados conseguiu-se visualizar a situação da cárie dentária, na faixa etária de doze anos no município, podendo propor medidas de prevenção e controle dessa doença.

Em Bonfim verificou-se o CPO-D de 4,78 , sendo alto quando comparado ao SB Brasil 2010 e SB Minas. Também não alcançou a meta proposta pela OMS $($ CPO-D $<1)$.

Diante desses dados verificou-se a necessidade de investimentos na área de saúde bucal, para assim melhorar a qualidade de vida desta população.

\section{6-BIBLIOGRAFIA}

1-ANTUNES, José Leopoldo Ferreira, PERES, Marco Aurélio. Epidemiologia da saúde bucal. Guanabara Koogan, Rio de Janeiro, 2006.
2- ATLAS DO DESENVOLVIMENTO HUMANO NO BRASIL. Rio de Janeiro, PNUD, IPEA, Fundação João Pinheiro, 2003. Disponível em: http://atlasbrasil.org.br/2013/pt/perfil_m/bonfim_mg. Acesso em: 26 maio de 2018

3- Brasil. Ministério da saúde. Portaria 1444 de 28 de dezembro de 2000. Estabelece incentivo financeiro para reorganização da saúde bucal prestada nos municípios por meio do Programa de Saúde da Família. Diário Oficial da União, Brasília, 138 (250): 85, 29 dez. 2000. Seção 1.

4- CARVALHO, Maria Célia Natel. DOLIVIEIRA, Sérgio Luiz. Saúde Bucal e ações preventivas: um estudo de caso em escolas públicas do município de Guarapuava. Eletron Lato Sensu, 2008.

5- CYPRIANO, Silvia. SOUZA, Maria da Luz Rosário de. WADA, Ronaldo Seichi. Avaliação de índices CPOD simplificados em levantamentos epidemiológicos de cárie dentária. Saúde Pública, São Paulo,v.39, n.2, p.285-292, 2005.

6-FIGUEIREDO, Rísia Maria de Oliveira. WASSAL, Thomaz. FLÓRIO, Flávia Martão. Frequência de impactos dos problemas de saúde bucal na qualidade de vida. RGO, Porto Alegre, v.45, n.1,p.11-16,2006.

7- GOMES, Patrícia Rodrigues. COSTA, Sandra Camanho. CYPRIANO, Silvia. SOUZA, Maria da Luz Rosário de. Paulínia, São Paulo Brasil: situação da cárie dentária com relação às metas OMS 2000 e 2010. Cad. Saúde Pública, Rio de Janeiro, v.20, n.3, p. 866-870, Rio de Janeiro.

8-LEITES, Antonio Cesar Bortowiski Rosa. PINTO, Márcia Bueno. SOUZA, Ezilmara Rolim de . Aspectos microbiológicos da cárie dental. Salusvita, v.25, n.2, p.135-48, 2006.

9-MELO, Tatiana Ribeiro de Campos. ANTUNES, José Leopoldo Ferreira. WALDMAN, Eliseu Alves. Áreas rurais: pólos de concentração de agravos à saúde bucal?, Arq Med, São Paulo,v.19,n.1-2, p. 6774,2005 .

10-Minas Gerais. Secretaria de Estado de Saúde. Subsecretaria de Políticas e Ações de Saúde. Superintendência de Redes de Atenção à Saúde. Diretoria de Saúde Bucal. SB Minas Gerais: pesquisa das condições de saúde bucal da população mineira: resultados principais. Belo Horizonte: SES-MG, 2013.

11-NARVAI,Paulo Capel et al. Cárie dentária no Brasil: declínio, iniquidade e exclusão social. Rev. Panam. Salud Publica, v.19,n.6, p.385-93,2006.

12-PERES, Marco Aurélio Et al. The association between socioeconomic development at the town level and distribuition of dental caries in Brazilian children. 
Rev panam. Salud publica, Kansas, v.14, n.3, p.149157,2003.

13- PINTO, Vitor Gomes. Saúde Bucal Coletiva. Editora Santos, Cap. 5, pág. 181-186, São Paulo, 2000.

14- RONCALLI, Angelo Giuseppe. CÔRTES, Maria Ilma de Souza. PERES, Karen Glazer. Perfis epidemiológicos de saúde bucal no Brasil e os modelos de vigilância. Cad. Saúde Pública, p.58-68, 2012.

15- SANTOS, Manuela Golvêa Campêlo dos. SANTOS, Ronaldo Campêlo dos. Fluoretação das Águas de Abastecimento Público no Combate à Cárie Dentária. Revista Brasileira de Ciências da Saúde,v.15, n.1, p.75-80, 2011. 